University of Wollongong

Research Online

Faculty of Informatics - Papers (Archive)

Faculty of Engineering and Information

Sciences

$1-1-2010$

\title{
Soliton steering by longitudinal modulation of the nonlinearity in waveguide arrays
}

Gaetano Assanto

University of Rome Tre

Luis A. Cisneros

Instituto Polite'cnico Nacional, Mexico

Antonmaria A. Minzoni

Universidad Nacional Autónoma de México

Benjamin D. Skuse

University of Edinburgh

Noel F. Smyth

University of Edinburgh

See next page for additional authors

Follow this and additional works at: https://ro.uow.edu.au/infopapers

Part of the Physical Sciences and Mathematics Commons

\section{Recommended Citation}

Assanto, Gaetano; Cisneros, Luis A.; Minzoni, Antonmaria A.; Skuse, Benjamin D.; Smyth, Noel F.; and Worthy, Annette L.: Soliton steering by longitudinal modulation of the nonlinearity in waveguide arrays 2010, 053903-1-053903-4.

https://ro.uow.edu.au/infopapers/1730

Research Online is the open access institutional repository for the University of Wollongong. For further information contact the UOW Library: research-pubs@uow.edu.au 


\title{
Soliton steering by longitudinal modulation of the nonlinearity in waveguide arrays
}

\author{
Abstract \\ We show how discrete solitary waves in one and two-dimensional waveguide arrays can be steered \\ across the lattice via the introduction of a longitudinal periodic modulation of the nonlinear response. \\ Through parametric energy transfer from the modulation to the solitary wave, the latter can increase its \\ width and overcome the Peierls-Nabarro potential to propagate freely.

\section{Keywords} \\ Soliton, steering, longitudinal, modulation, nonlinearity, waveguide, arrays

\section{Disciplines} \\ Physical Sciences and Mathematics

\section{Publication Details} \\ Assanto, G., Cisneros, L., Minzoni, A., Skuse, B. D., Smyth, N. F. \& Worthy, A. L. (2010). Soliton steering by \\ longitudinal modulation of the nonlinearity in waveguide arrays. Physical Review Letters, 104 (5), \\ 053903-1-053903-4.
}

\section{Authors}

Gaetano Assanto, Luis A. Cisneros, Antonmaria A. Minzoni, Benjamin D. Skuse, Noel F. Smyth, and Annette L. Worthy 


\title{
Soliton Steering by Longitudinal Modulation of the Nonlinearity in Waveguide Arrays
}

\author{
Gaetano Assanto, ${ }^{1}$ Luis A. Cisneros, ${ }^{2}$ Antonmaria A. Minzoni, ${ }^{3}$ Benjamin D. Skuse, ${ }^{4}$ \\ Noel F. Smyth, ${ }^{4}$ and Annette L. Worthy ${ }^{5}$ \\ ${ }^{1}$ NooEL-Nonlinear Optics and OptoElectronics Lab, Department of Electronic Engineering, University of Rome "Roma Tre," \\ Via della Vasca Navale 84, 00146 Rome, Italy \\ ${ }^{2}$ Escuela Superior de Física y Matemáticas, Instituto Politécnico Nacional, Boulevard Adolfo Lopez Mateos, México D.F., México \\ ${ }^{3}$ Fenomenos Nonlineales y Mecánica (FENOMEC), Department of Mathematics and Mechanics, \\ Instituto de Investigación en Matemáticas Aplicadas y Sistemas, Universidad Nacional Autónoma de México, \\ 01000 México D.F., México \\ ${ }^{4}$ School of Mathematics and Maxwell Institute for Mathematical Sciences, The King's Buildings, University of Edinburgh, \\ Edinburgh, Scotland, United Kingdom, EH9 3JZ \\ ${ }^{5}$ School of Mathematics and Applied Statistics, University of Wollongong, Wollongong, New South Wales 2522, Australia
} (Received 18 December 2009; published 5 February 2010)

\begin{abstract}
We show how discrete solitary waves in one and two-dimensional waveguide arrays can be steered across the lattice via the introduction of a longitudinal periodic modulation of the nonlinear response. Through parametric energy transfer from the modulation to the solitary wave, the latter can increase its width and overcome the Peierls-Nabarro potential to propagate freely.
\end{abstract}

Discrete nonlinear propagation, initially introduced by Fermi, Pasta, and Ulam [1] with their numerical experiments on chains of discrete particles under the influence of nonlinear nearest neighbor coupling forces, has been dealt with in mechanical and biological systems, as well as in optics, mathematics, Josephson junctions, and BoseEinstein condensates [1-10]. In optical physics, nonlinear waveguide arrays and discrete lattices in one and two dimensions have received a great deal of attention in terms of both fundamental and applied aspects of light localization and interactions in systems with transversely modulated properties $[4,11,12]$. Discrete optical solitons and their propagation in dielectric or semiconductor arrays are considered promising avenues towards self-routing and signal steering for spatial demultiplexing. A number of approaches have been investigated to exploit discrete arrays and their unique properties, including the introduction of defects, interaction of discrete solitons with other solitons or signals, etc., as well as the use of Bragg gratings [12], Zener tunneling [13-15], linearly induced resonances, and linear modulation to achieve energy transfer between solitary waves in different bands [16-19]. In this Letter we investigate the physics of an array in the presence of a periodic modulation of the nonlinearity along the direction of propagation. Since solitary waves are pinned to the lattice due to the Peierls-Nabarro (PN) potential $[20,21]$, we show how a parametric resonance modifies the potential, resulting in the free motion of a solitary wave across the lattice. As a solitary wave has an internal frequency it can resonantly interact with the periodic nonlinearity modulation, producing an amplitude and width oscillation of relatively large amplitude which modifies the potential. It is known in other oscillating systems that subharmonic resonances produce an exponential transfer of energy [22]. We demonstrate that parametric forcing at twice the oscillation frequency of the solitary wave is extremely effective in transferring energy to the latter; the solitary wave increases its width, which in turn reduces the PN potential, resulting in its untrapping and propagation with a transverse velocity. Different frequencies for the spatial modulation along the propagation direction can be used to position the solitary wave at a prescribed location in the array. Let us first consider an infinitely extended one-dimensional (1D) array of weakly coupled single mode channel waveguides. The coupled mode equations for the amplitudes $Q_{n}$ of the eigensolutions in the periodic channels can be cast in a nondimensional form [23,24], which in the local regime reduce to the discrete nonlinear Schrödinger (DNLS) equation

$$
i \dot{Q}_{n}+\frac{1}{2}\left(Q_{n+1}-2 Q_{n}+Q_{n-1}\right)+\alpha(z)\left|Q_{n}\right|^{2} Q_{n}=0,
$$

where the dot denotes the derivative with respect to $z$. The periodic function $\alpha(z)$ arises from the periodic modulation along the propagation direction $\alpha=1+q \cos \mu z$, with adjustable parameters $q$ and $\mu$. Similarly, for a twodimensional array the two-dimensional (2D) DNLS equation in the local limit is

$$
\begin{aligned}
& i \dot{Q}_{m, n}+\frac{1}{2}(\left.Q_{m+1, n}+Q_{m-1, n}+Q_{m, n+1}+Q_{m, n-1}\right) \\
&-2 Q_{m, n}+(1+q \cos \mu z)\left|Q_{m, n}\right|^{2} Q_{m, n}=0 .
\end{aligned}
$$

The discrete equations (1) and (2) contain a periodic modulation in the nonlinear terms, with the linear terms constant. A periodic modulation of the nonlinearity along the propagation direction $z$ has quite a different role on the physics of the interaction as compared to previous work carried out with modulation of the linear properties of the array [12,17-19], the latter giving rise to momentum trans- 
fer between the lattice sites. In the present case the parametric excitation does not produce transfer of transverse momentum; conversely, it widens the soliton eigensolution, resulting in its untrapping from the lattice.

To better understand and model the effect of the parametric resonance modulation, solutions of the DNLS equations are constructed using an averaged Lagrangian based on suitable trial functions $[25,26]$. Such a suitable trial function in one dimension is

$$
Q_{n}=\operatorname{asech} \frac{n-\zeta(z)}{w} e^{i \sigma(z)+i V(n-\zeta)}+i g e^{i \sigma(z)+i V(n-\zeta)} .
$$

The variable $\sigma$ is the phase, with the $z$ derivative $\dot{\sigma}$ giving the local frequency of the solitary wave. The variable $g$ represents the height of the shelf of diffractive radiation, visible in Fig. 3, under the solitary wave [26]. This shelf has width $\ell$ and $g$ is nonzero in $\zeta-\ell / 2<n<\zeta+\ell / 2$. The width $\ell$ will be determined later. The modulation equations, obtained as variational equations from the averaged Lagrangian, provide the evolution equations for the discrete solitary wave as $[25,26]$

$$
\begin{gathered}
\frac{d}{d z}\left(2 a^{2} w+\ell g^{2}\right)=-\frac{16 \delta r^{2}}{3 \hat{a}}, \\
\frac{d}{d z}(a w)=\frac{\ell g}{\pi}\left[a^{2}(1+q \cos \mu z)-\frac{1}{2 w^{2}}\right], \\
\frac{d g}{d z}=\frac{2 a}{3 \pi}\left[\frac{1}{w^{2}}-(1+q \cos \mu z) a^{2}\right]-2 \delta g, \\
\frac{d \zeta}{d z}=V, \\
\frac{d}{d z}\left(2 a^{2} w+\ell g^{2}\right) V=-\frac{(2 \pi)^{5}}{6} a^{4} w^{4} e^{-\beta \pi^{2} w} \sin 2 \pi \zeta .
\end{gathered}
$$

The lattice effect is captured to this order by the periodic term in the momentum Eq. (8). This is the Peierls-Nabarro potential, which becomes smaller as the width $w$ of the solitary wave increases. The damping term $2 \delta g$ takes into account the effect of the diffractive radiation shed by the solitary wave as it evolves. Assuming a small (transverse) velocity for the solitary wave, we use the same radiation damping term as in Kath and Smyth, even though it was derived there for a static solitary wave or one moving with a constant velocity [26]. Then

$$
\delta=\frac{3 r}{8 r_{0} \sqrt{\pi z}}, \quad r^{2}=\ell^{-1}\left(2 a^{2} w+\ell g^{2}-2 \hat{a}^{2} \hat{w}\right),
$$

where the subscript 0 refers to values at $z=0$ and the caret refers to fixed point values. The solution of the modulation equations can be qualitatively described as follows. In the absence of parametric forcing, so that $q=0$, the modulation equations have a fixed point which is a center of constant frequency $\dot{\sigma}$, with $\ell=3 \pi^{2} /(8 \hat{a})$ [26]. In this case Eqs. (7) and (8) for the position of the solitary wave have stable fixed points at the minima of the PeierlsNabarro potential in the momentum equation (8). This implies that solitary waves with sufficiently small initial velocity can be trapped, provided that they are narrow $(w \ll 1)$. The strongest forcing occurs in the frequency band for $\mu$ centered around $2 \dot{\sigma}$, where $\dot{\sigma}$ is the constant fixed point frequency. In this band the solitary wave width $w$ tends to increase and is stabilized through radiative losses and the effect of nonlinearity. As $w$ increases, the solitary wave becomes wider and the periodic potential smaller, until the self-confined wave can eventually escape and travel like a continuum solitary wave. We stress that this mechanism for escaping is different from that driven by momentum transfer to the lattice, as studied in previous work [12,17-19]. This predicted behavior was verified in numerical experiments. Away from the subharmonic resonance the solitary wave is trapped in the lattice and undergoes an amplitude and width oscillation, as illustrated in Fig. 1 . The power $a^{2} w$ is slowly decreasing due to the large amount of diffractive radiation shed. Figure 2 shows the solitary wave position for the case of subharmonic resonance. From Fig. 3 it is apparent that the solitary wave leaks a considerable amount of radiation as it evolves in the plane of the array, in particular, it sheds a large amount of diffractive radiation at the points at which it starts to move from site to site. As the solitary wave is trapped for $\mu$ small, this proves that the subharmonic resonance with $\mu=0.8$ enables the discrete solitary wave to escape the trapping potential and propagate across the lattice, a behavior unavailable with $q=0$. As the shed radiation and solitary wave interact, the latter changes shape, which in turn changes the PN potential by reducing the effective width of the solitary wave in the PN exponent, in a similar fashion to other moving solitary wave problems [27]. To obtain a good initial agreement with the numerical solution, the modulation equations need be adjusted to take into account the changing Peierls-Nabarro potential by tuning

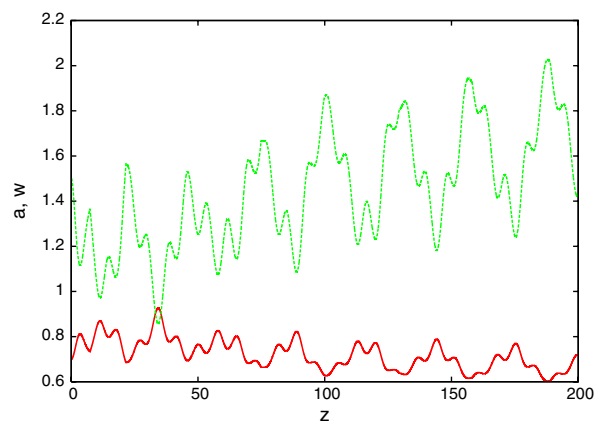

FIG. 1 (color online). Solution of DNLS equation (1) for the amplitude $a$ (solid line, red) and width $w$ (dashed line, green) of the solitary wave as given by the numerical solution for the initial conditions $a=0.7, w=1.5$, and $V=0.1$ with $q=0.15$ and $\mu=0.55$. 


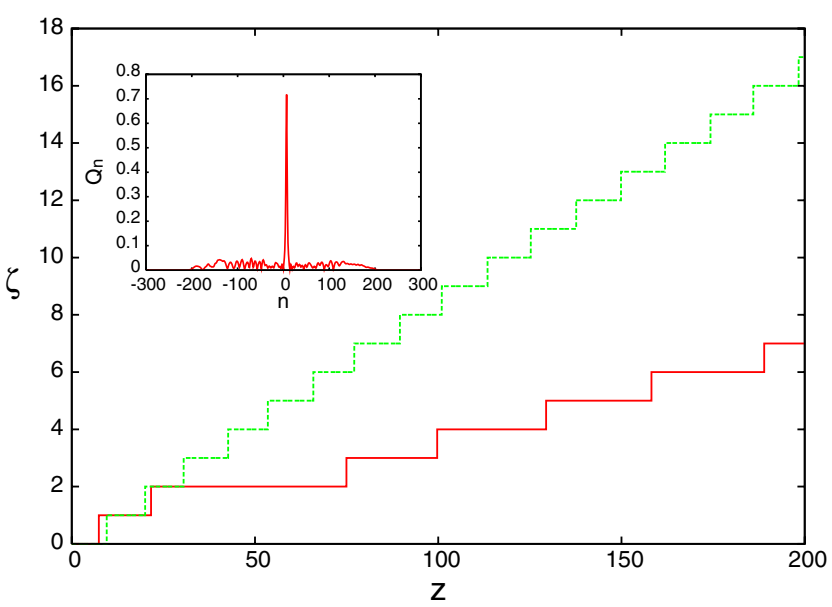

FIG. 2 (color online). Position $\zeta$ of the soliton from the DNLS equation (1) for the initial conditions $a=0.7, w=1.5$, and $V=$ 0.1 with $q=0.15$ and $\mu=0.8$. Numerical solution: solid line (red); solution of modulation equations: dashed line (green). The inset shows the solution for $\left|Q_{n}\right|$ at $z=200$.

the parameter $\beta$-which from the pure modulation analysis should be equal to 1 . Good agreement was obtained for $\beta=0.98$, demonstrating that the trapping behavior is sensitive to the Peierls-Nabarro potential. The position agreement is quite good until around $z \approx 30$. Afterwards the solutions diverge, because the shed diffractive radiation carries away momentum, which is not accounted for in the modulation equations. The inclusion of shed radiation momentum is a nontrivial problem as it involves the solution of a moving boundary value problem with unknown, accelerating boundaries [28]. In any event, both the numerical and modulation equation results provide the same qualitative picture of a discrete solitary wave which the parametric resonance has unlocked from the PN potential.

Finally, Fig. 4 shows the case of an escaping solitary wave at a simple $1: 1$ resonance $\mu=\dot{\sigma}$. The important

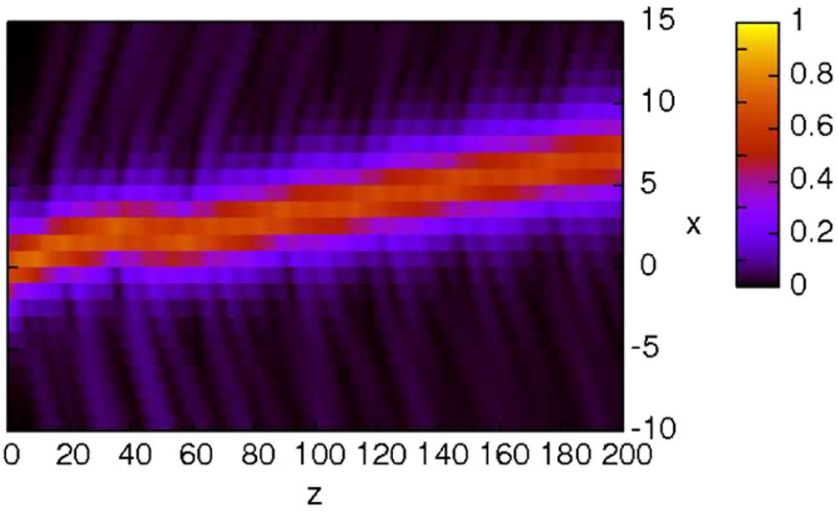

FIG. 3 (color online). Evolution in $x z$ of the solution of the DNLS equations (1) for the initial conditions $a=0.7, w=1.5$, and $V=0.1$ with $q=0.15$ and $\mu=0.8$. difference to the case of subharmonic resonance is that much higher initial velocities are required for escape.

The equivalent situation in two spatial transverse dimensions can be studied along the same lines, with the input beam taken as a product of the one-dimensional trial functions (3) and $\exp \left(-n^{2} / w^{2}\right)$ in the $n$ coordinate

$$
Q_{n, m}=a e^{-(m-\zeta)^{2} / w^{2}-n^{2} / w^{2}} e^{i \sigma+i(m-\zeta) V}+i g e^{i \sigma+i(m-\zeta) V} .
$$

The resulting modulation equations are

$$
\begin{gathered}
\pi \frac{d}{d z} a w^{2}=\Lambda \dot{\sigma} g, \quad \frac{d}{d z}\left(\pi a^{2} w^{2}+\Lambda g^{2}\right)=0, \\
\frac{d g}{d z}=a\left[w^{-2} e^{-1 /\left(2 w^{2}\right)}-\frac{a^{2}}{4}(1+q \cos \mu z)\right], \\
\frac{d \sigma}{d z}=2 w^{-2} e^{-1 /\left(2 w^{2}\right)}+e^{-1 /\left(2 w^{2}\right)}-2, \quad \frac{d \zeta}{d z}=V, \\
\frac{d}{d z}\left(\pi a^{2} w^{2}+\Lambda g^{2}\right) V=\frac{(2 \pi)^{6}}{3 \pi} a^{4} w^{5} e^{-\pi^{2} w} \sin 2 \pi \zeta .
\end{gathered}
$$

Here

$$
\Lambda=\frac{3}{2} \frac{e^{-1 /\left(2 w^{2}\right)}}{w^{3}\left[2-2 w^{-2} e^{-1 /\left(2 w^{2}\right)}-e^{-1 /\left(2 w^{2}\right)}\right]} .
$$

It should be stressed that the discreteness in the lattice is captured by the $\exp \left[-1 /\left(2 w^{2}\right)\right]$ term. This is responsible for the arrest of the collapse of solitary waves in the continuous two space dimensional nonlinear Schrödinger (NLS) equation, since it increases as $w$ becomes smaller, transforming the degenerate fixed point of the continuum NLS equation into a stable center for the discrete equivalent. The modulation equations are solved in a parameter regime differing from that considered by Arevalo [29], who considered the mobility of discrete solitary waves and vortices. In his work the solitary wave amplitudes

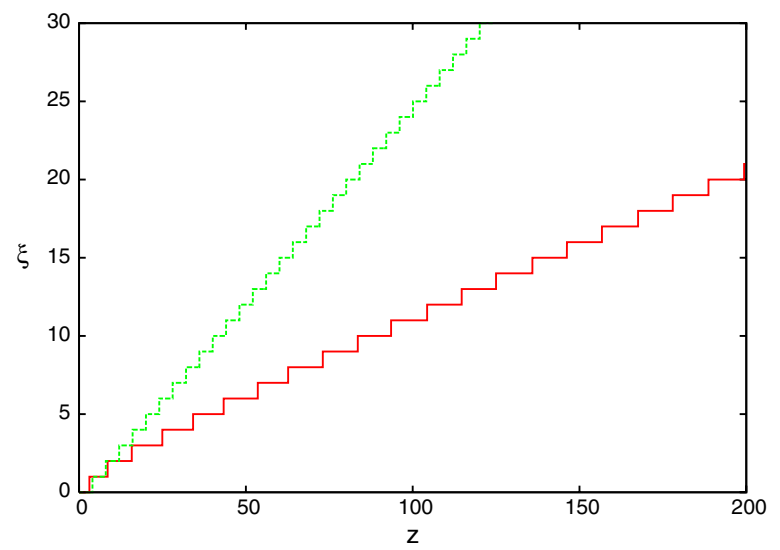

FIG. 4 (color online). Position $\zeta$ for the DNLS equation (1) for the initial conditions $a=0.7, w=1.5$, and $V=0.25$ with $q=$ 0.15 and $\mu=0.38$. Numerical: solid line (red); modulation: dashed line (green). 


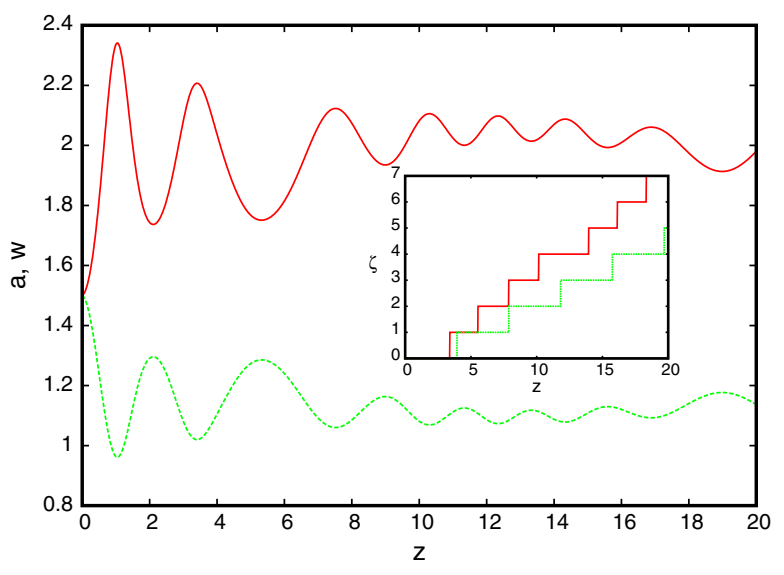

FIG. 5 (color online). Solution of $2+1$ dimensional DNLS equation (2) for the initial conditions $a=1.5, w=1.5$, and $V=$ 0.25 with $q=0.18$. Numerical amplitude $a$ (solid line, red) and width $w$ (dashed line, green) for $\mu=0.5$. Inset: position $\zeta$ for $\mu=1.4$. Numerical solution: solid line (red); solution of modulation equations: dashed line (green).

were taken to be small and the widths relatively large. In the present work we consider narrow solitary waves with larger amplitudes in order to study the effect of the PeierlsNabarro potential, which was exponentially small in Arevalo's parameter regime [29].

Figure 5 shows the solitary wave transverse position in the case of escape due to a subharmonic resonance. This escape case is qualitatively the same as the equivalent onedimensional case of Fig. 2. Figure 6 displays a twodimensional plot of the numerical solution at $z=10$ : the large amount of shed diffractive radiation is clearly visible. For small $\mu$ there is no escape from the potential.

We have introduced a parametric resonance mechanism which allows discrete solitary waves in waveguide arrays to escape the Peierls-Nabarro potential by means of a longitudinal modulation of the nonlinearity. Numerical solutions and an asymptotic analysis are in good agreement, despite the latter not fully accounting for the momentum of the shed diffractive radiation. The parametric resonance is very effective in producing amplitude and

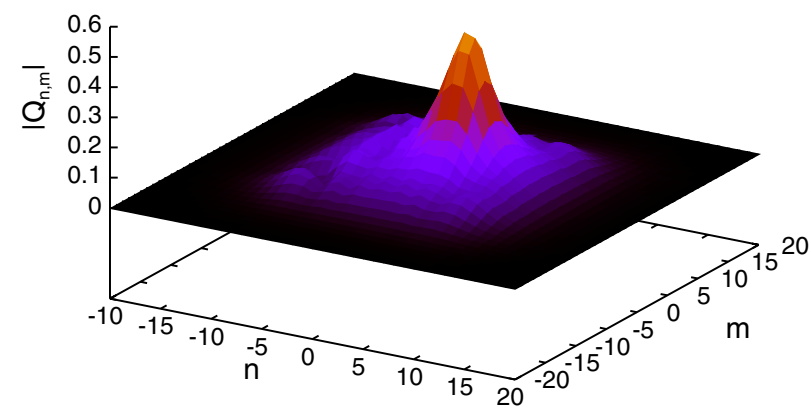

FIG. 6 (color online). Solution of $2+1$ dimensional DNLS equation (2) for $\left|Q_{m, n}\right|$ at $z=10$ for the initial conditions $a=$ $1.5, w=1.5$, and $V=0.25$ with $\mu=1.4$ and $q=0.18$. width oscillations in very slow solitary waves; such oscillations increase the soliton width and reduce the PeierlsNabarro potential, eventually unlocking discrete solitons and letting them propagate with a finite transverse velocity across the lattice. We investigated this novel mechanism in both one- and two-dimensional arrays.

The authors would like to thank the referees for useful comments which greatly improved the manuscript. This research was supported by the Royal Society of London under Grant No. JP090179.

[1] E. Fermi, J. Pasta, and S. Ulam, Los Alamos Report No. LA-1940, 1955.

[2] A. S. Davydov, J. Theor. Biol. 38, 559 (1973).

[3] G. Careri et al., Phys. Rev. B 30, 4689 (1984).

[4] F. Lederer et al., Phys. Rep. 463, 1 (2008).

[5] J. C. Eilbeck, P. S. Lomdhal, and A.C. Scott, Physica (Amsterdam) 16D, 318 (1985).

[6] A. C. Scott, Phys. Rev. A 26, 578 (1982).

[7] F. Calogero, J. Math. Phys. (N.Y.) 12, 419 (1971).

[8] E. Trias, J. J. Mazo, and T. P. Orlando, Phys. Rev. Lett. 84, 741 (2000).

[9] A. J. Sievers and S. Takeno, Phys. Rev. Lett. 61, 970 (1988).

[10] A. Trombettoni and A. Smerzi, Phys. Rev. Lett. 86, 2353 (2001).

[11] A. Fratalocchi and G. Assanto, Phys. Rev. A 75, 063828 (2007).

[12] A. A. Sukhorukov and Y. S. Kivshar, Phys. Rev. Lett. 97, 233901 (2006).

[13] R. Khomeriki and S. Ruffo, Phys. Rev. Lett. 94, 113904 (2005).

[14] H. Trompeter et al., Phys. Rev. Lett. 96, 053903 (2006).

[15] A. Fratalocchi and G. Assanto, K. A. Brzdakiewicz, and M. A. Karpierz, Opt. Express 14, 2021 (2006).

[16] C. R. Rosberg et al., Opt. Lett. 31, 1498 (2006).

[17] A. Szameit et al., Phys. Rev. Lett. 102, 153901 (2009).

[18] K. G. Makris, D. N. Christodoulides, O. Peleg, M. Segev, and D. Kip, Opt. Express 16, 10309 (2008).

[19] K. Shandarova et al., Phys. Rev. Lett. 102, 123905 (2009).

[20] R. Peierls, Proc. Phys. Soc. London 52, 34 (1940).

[21] F. R. N. Nabarro, Proc. Phys. Soc. London 59, 256 (1947).

[22] J. D. Cole and J. K. Kevorkian, Perturbation Methods in Applied Mathematics (Springer-Verlag, Berlin, 1981).

[23] A. Fratalocchi and G. Assanto, Phys. Rev. E 72, 066608 (2005).

[24] A. Fratalocchi and G. Assanto, Phys. Rev. A 76, 042108 (2007).

[25] A. A. Minzoni, N. F. Smyth, and A. L. Worthy, J. Opt. Soc. Am. B 24, 1549 (2007).

[26] W. L. Kath and N. F. Smyth, Phys. Rev. E 51, 1484 (1995).

[27] L. Cisneros and A. A. Minzoni, Physica (Amsterdam) 237D, 50 (2008).

[28] N.F. Smyth and W.L. Kath, Phys. Rev. E 63, 036614 (2001).

[29] E. Arevalo, Phys. Rev. Lett. 102, 224102 (2009). 